\title{
Properties of State Estimation for Power System with Quadrature Booster in Rectangular Coordinates
}

\author{
Tomasz Okon, Kazimierz Wilkosz \\ Department of Electrical Power Engineering, Wroclaw University of Science and Technology, \\ Wybrzeze Wyspianskiego 27, Wroclaw, Poland \\ tomasz.okon@pwr.edu.pl
}

\begin{abstract}
The paper presents a state estimation of a power system with a phase shifter. A new versatile model of the phase shifter for steady state analyses is introduced. The mentioned model allows for consideration of different types of phase shifters. In the paper, original investigations of properties of the state estimation for a power system with a quadrature booster (to be one of types of phase shifters) are performed. The state estimation is considered in the rectangular coordinate system. During the investigations, impact of data redundancy on such parameters of the state estimation as: the number of iterations, a condition number of a coefficient matrix, parameters characterizing accuracy of calculation results are taken into account. At the end of the paper, conclusions from the conducted investigations are presented.
\end{abstract}

Index Terms-Phase shifter; State estimation; Power system.

\section{INTRODUCTION}

Nowadays, phase shifters are more and more frequently used in power systems for power flow control. Their immanent property is possibility of control of phase angles of voltages in power systems. Changing the phase shift between terminal voltage phasors, the phase shifter regulates power flow through a power line [1]-[5]. In consequence, the phase shifter changes power flows in a power system, and it can lead to elimination of undesirable power flows.

A model of a power system influences calculations made for this system, in particular, state-estimation calculations [6] enabling to obtain the reliable estimate of the powersystem state vector. The paper is devoted presentation of results of original investigations on influence of occurrence of the phase shifter in a power system on properties of state estimation. The considered type of the phase shifter is a quadrature booster [7]-[9]. It is one of the simplest phase shifters. The quadrature booster derives a voltage from the supply that is phase-shifted by $90^{\circ}$ and re-applied to the voltage before it. In this way one forms the voltage at the beginning of a power line, of which phase is different than the phase of the voltage before the phase shifter.

The paper considers the power-system state estimation in a rectangular coordinate system. In general, properties of the power-system state estimation in this coordinate system are better than in a polar coordinate system [10], [11].
In the paper, the quadrature booster is modeled using two real voltage sources. One of them is in a shunt branch and second one is in a series branch. The assumed model is other than models of phase shifters that can be found in other papers [2], [3], [8], [9], [12]-[20]. The here-considered model can be used for modeling different types of phase shifters, otherwise than models from the earlier-mentioned papers.

In the further part of the paper, the considered method for the power-system state estimation and indices characterizing properties of the estimation method are presented. Next, a model of the quadrature booster is formulated. Main part of the paper is description of investigations, whose aim is to show differences between properties of the state estimation for a power system with the quadrature booster and without this device. At the end, the most important conclusions from the conducted investigations are given.

\section{CONSIDERED Method FOR POWER System StATE ESTIMATION}

\section{A. Objective Function}

In the paper, the weighted least squares power-system state estimation method is considered. For that method, an objective function is following [10]

$$
J(\mathbf{x})=\frac{1}{2}[\mathbf{z}-\mathbf{h}(\mathbf{x})]^{\mathbf{T}} \mathbf{R}^{-1}[\mathbf{z}-\mathbf{h}(\mathbf{x})]
$$

where $\mathbf{x}$ is a power system state vector; $\mathbf{z}$ is a vector of measurements; $\mathbf{h}(\mathbf{x})$ is a vector of functions (also nonlinear) of vector $\mathbf{x}$, representing dependence of measured quantities on the state vector; $\mathbf{R}$ is a diagonal matrix of measurement covariances.

State vector $\mathbf{x}$ in the rectangular coordinate system is defined as

$$
\mathbf{x}=\left[e_{1}, e_{2}, \ldots, e_{n}, f_{2}, f_{3}, \ldots, f_{n}\right]^{\mathrm{T}}
$$

where $e_{i} i=1,2, \ldots, n$ are real parts of voltages at the buses $1,2, \ldots, n ; f_{i} i=2,3, \ldots, n$ are imaginary parts of voltages at the buses $2,3, \ldots, n$.

The relationships among measured quantities and elements of the state vector are as follows [11]: 


$$
\begin{aligned}
& V_{i}=V_{i}, \\
& P_{i}-j Q_{i}=\overline{\mathbf{V}}_{i}^{*} \mathbf{Y}_{\text {row } i} \mathbf{V} \\
& P_{i j}-j Q_{i j}=\left[\begin{array}{ll}
-\left(\overline{\mathbf{y}}_{s i}+\overline{\mathbf{y}}_{i j}\right) & \overline{\mathbf{y}}_{i j}
\end{array}\right] \times\left[\begin{array}{ll}
V_{i}^{2} & \overline{\mathbf{V}}_{j} \times \overline{\mathbf{V}}_{i}^{*}
\end{array}\right]^{T},
\end{aligned}
$$

where $V_{i}$ is a voltage magnitude at $i$-th bus; $P_{i}, Q_{i}$ are an active and reactive power injection at $i$-th bus, respectively; $P_{i j} Q_{i j}$ are an active and reactive power flow, respectively, between $i$-th and $j$-th bus, measured at $i$-th bus; $\overline{\mathbf{V}}_{i}$ is a voltage at $i$-th bus; $\overline{\mathbf{y}}_{i j}$ is an admittance of the series branch connecting $i$-th and $j$-th bus; $\overline{\mathbf{y}}_{s i}$ is an admittance of the shunt branch at $i$-th bus; $\mathbf{Y}_{\text {row } i}$ is $i$-th row of an admittance matrix

$$
\mathbf{Y}_{\text {row } i}=\left[\overline{\mathbf{Y}}_{i 1}, \overline{\mathbf{Y}}_{i 2}, \ldots \overline{\mathbf{Y}}_{i n}\right]
$$

where $\overline{\mathbf{Y}}_{i k} i=1,2, \ldots, n$ are elements of the admittance matrix; $\mathbf{V}$ is a vector

$$
\mathbf{V}=\left[\overline{\mathbf{V}}_{1}, \overline{\mathbf{V}}_{2}, \ldots \overline{\mathbf{V}}_{n}\right]^{\mathrm{T}}
$$

The relationships (3)-(5) are base for determination of elements of function vector $\mathbf{h}(\mathbf{x})$.

\section{B. Method of Solution of State Estimation Problem}

A solution of the state-estimation problem is achieved by solving the normal-equation set

$$
\mathbf{G}\left(\mathbf{x}^{k}\right) \times\left(\mathbf{x}^{k+1}-\mathbf{x}^{k}\right)=-\mathbf{g}\left(\mathbf{x}^{k}\right),
$$

where $k$ is a number of iteration, $\mathbf{x}^{k}$ is a solution vector at $k$-th iteration:

$$
\begin{gathered}
\mathbf{G}\left(\mathbf{x}^{k}\right)=\mathbf{H}^{T}\left(\mathbf{x}^{k}\right) \times \mathbf{R}^{-1} \times \mathbf{H}\left(\mathbf{x}^{k}\right), \\
\mathbf{H}(\mathbf{x})=\frac{\partial \mathbf{h}(\mathbf{x})}{\partial \mathbf{x}}, \\
\mathbf{g}(\mathbf{x})=\frac{\partial J(\mathbf{x})}{\partial \mathbf{x}}=-\mathbf{H}^{T}(\mathbf{x}) \mathbf{R}^{-1}[\mathbf{z}-\mathbf{h}(\mathbf{x})],
\end{gathered}
$$

where $\mathbf{G}(\mathbf{x})$ is called a gain matrix. It is a symmetric, sparse, and positive determined matrix for a fully observable power system.

\section{INDICES CHARACTERIZED PROPERTIES OF POWER SYSTEM STATE ESTIMATION}

The following indices are used to characterize properties of power-system state estimation:

- number of iterations in a state-estimation process,

- a condition number of the gain matrix,

- ratio $J_{e} / J_{M}$.

Number of iterations in a state estimation process affects the time of calculations. It depends on the convergence of a calculation process.
The condition number of the gain matrix $\mathbf{G}$ is a measure of conditioning of a state-estimation process [21]. In the paper, one assumes that its definition is following

$$
\operatorname{cond}(\mathbf{G})=\frac{\left|\lambda_{M}\right|}{\left|\lambda_{m}\right|},
$$

where $\lambda_{m}, \lambda_{M}$ are the minimal and maximal (by moduli) eigenvalues of $\mathbf{G}$ matrix, respectively.

The condition number is larger, the conditioning of the estimation process is worse. The ill-conditioning of the estimation process often leads to a worse convergence of the process or even to lack of the convergence of this process.

Ratio $J_{e} / J_{M}$ [22]:

$$
\left\{\begin{array}{l}
J_{M}=\frac{1}{m} \sum_{i=1}^{m}\left[\left(z_{i}-z_{i}^{r}\right) / \sigma_{i}\right]^{2}, \\
J_{e}=\frac{1}{m} \sum_{i=1}^{m}\left[\left(\hat{z}_{i}-z_{i}^{r}\right) / \sigma_{i}\right]^{2},
\end{array}\right.
$$

where $z_{i}, \hat{z}_{i}, z_{i}^{r}$ are the measured, estimated and real value of $i$-th measured quantity, respectively; $\sigma_{i}$ is a variance of the measurement of $i$-th quantity; $m$ is a number of the measured quantities; characterizes accuracy of a state estimation.

Ratio $J_{e} / J_{M}$ should satisfy the condition

$$
\frac{J_{e}}{J_{M}}<1
$$

If condition (14)is not satisfied, then accuracy of results of estimation is assessed as insufficient.

\section{QUADRATURE BOOSTER}

In general, an equivalent circuit of the phase shifter can be presented as it is in Fig. 1. In the equivalent circuit, there are distinguished: a shunt Excitation Transformer (ET) usually with delta connected primary windings, a series Boosting Transformer (BT), which injects a series voltage ( $\overline{\mathbf{V}}_{\mathrm{BT}}$ ) in a power system, and a tap changer. The phasor of the series voltage ( $\left.\overline{\mathbf{V}}_{\mathrm{BT}}\right)$ is controlled by the tap changer. In the conventional phase shifter, mechanical switches are usually embedded with the ET transformer. Replacement of mechanical switches by semiconductor switches enable to overcome the following disadvantages of the conventional phase shifter [2]:

- high response time as result of inertia of moving parts,

- high level of maintenances due to mechanical contacts and oil deterioration.

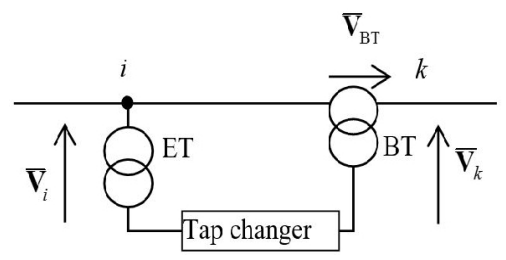

Fig. 1. An equivalent circuit of the phase shifter. 
The quadrature booster is a type of the phase shifter which allows obtaining the phase shift between voltage $\overline{\mathbf{V}}_{\text {BT }}$ and voltage $\overline{\mathbf{V}}_{i}$ equal to $-90^{\circ}$ or $90^{\circ}$.

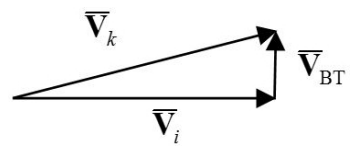

Fig. 2. Phasor diagram for the quadrature booster.

In the paper, it is assumed, that the quadrature booster is represented by the model to be shown in Fig. 3. The model consists of two controllable voltage sources. Their internal impedances $\overline{\mathbf{z}}_{\text {ET }}$ and $\overline{\mathbf{z}}_{\mathrm{BT}}$ represent impedances of transformers ET and BT, respectively.

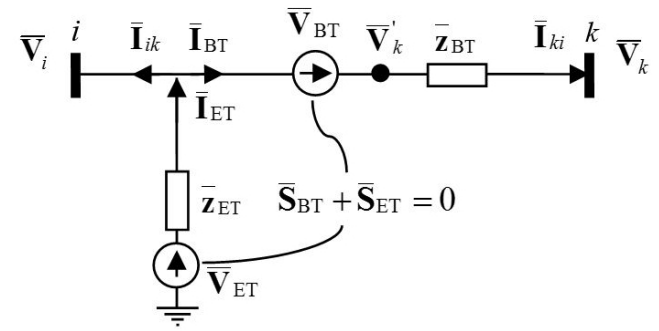

Fig. 3. The assumed model for the quadrature booster.

For the assumed model, the following fundamental nodal equations can be derived:

$$
\begin{gathered}
\overline{\mathbf{S}}_{i k}^{*}=-V_{i}^{2}\left(\overline{\mathbf{y}}_{\mathrm{ET}}+\overline{\mathbf{y}}_{\mathrm{BT}}\right)+ \\
+\overline{\mathbf{V}}_{k} \overline{\mathbf{V}}_{i}^{*} \overline{\mathbf{y}}_{\mathrm{BT}}-\overline{\mathbf{V}}_{\mathrm{BT}} \overline{\mathbf{V}}_{i}^{*-} \overline{\mathbf{y}}_{\mathrm{BT}}+\overline{\mathbf{V}}_{\mathrm{ET}} \overline{\mathbf{V}}_{i}^{*} \overline{\mathbf{y}}_{\mathrm{BT}}, \\
\overline{\mathbf{S}}_{k i}^{*}=-V_{k}^{2} \overline{\mathbf{y}}_{\mathrm{BT}}+\overline{\mathbf{V}}_{i} \overline{\mathbf{V}}_{k}^{*} \overline{\mathbf{y}}_{\mathrm{BT}}+\overline{\mathbf{V}}_{\mathrm{BT}} \overline{\mathbf{V}}_{k}^{*} \overline{\mathbf{y}}_{\mathrm{BT}}, \\
\overline{\mathbf{S}}_{\mathrm{BT}}^{*}=V_{\mathrm{BT}}^{2} \overline{\mathbf{y}}_{\mathrm{BT}}+\overline{\mathbf{V}}_{i} \overline{\mathbf{V}}_{\mathrm{BT}}^{*} \overline{\mathbf{y}}_{\mathrm{BT}}-\overline{\mathbf{V}}_{k} \overline{\mathbf{V}}_{\mathrm{BT}}^{*} \overline{\mathbf{y}}_{\mathrm{BT}}, \\
\overline{\mathbf{S}}_{\mathrm{ET}}^{*}=V_{\mathrm{ET}}^{2} \overline{\mathbf{y}}_{\mathrm{ET}}-\overline{\mathbf{V}}_{i} \overline{\mathbf{V}}_{\mathrm{ET}}^{*} \overline{\mathbf{y}}_{\mathrm{ET}} .
\end{gathered}
$$

where $\overline{\mathbf{y}}_{\mathrm{ET}}=1 / \overline{\mathbf{z}}_{\mathrm{ET}}, \overline{\mathbf{y}}_{\mathrm{BT}}=1 / \overline{\mathbf{z}}_{\mathrm{BT}}, V_{\mathrm{ET}}, \quad V_{\mathrm{BT}}$ are voltage magnitudes of $\overline{\mathbf{V}}_{\mathrm{ET}}$ and $\overline{\mathbf{V}}_{\mathrm{BT}}$, respectively.

Neglecting phase-shifter losses, we can state that the phase shifter cannot absorb and injects complex power, i.e. the complex power supplied to the excited transformer equals to the complex power demanded by the boosting transformer

$$
\overline{\mathbf{S}}_{\mathrm{ET}}+\overline{\mathbf{S}}_{\mathrm{BT}}=0 \text {. }
$$

Bus powers $\overline{\mathbf{S}}_{i-\mathrm{AC}}$ and $\overline{\mathbf{S}}_{k-\mathrm{AC}}$, calculated from (4), are updated in the following way:

$$
\begin{aligned}
\overline{\mathbf{S}}_{i} & =\overline{\mathbf{S}}_{i-\mathrm{AC}}-\overline{\mathbf{S}}_{i k}, \\
\overline{\mathbf{S}}_{k} & =\overline{\mathbf{S}}_{k-\mathrm{AC}}-\overline{\mathbf{S}}_{k i} .
\end{aligned}
$$

Equations (15)-(21) concern the phase shifter which can injects $\overline{\mathbf{V}}_{\mathrm{BT}}$ with any phase angle. If PST is quadrature booster the following assumptions must be taken into account:

- For the polar coordinate system

$$
\delta_{\mathrm{BT}}-\delta_{i}-\delta_{\mathrm{BT}}^{\mathrm{tar}}=0
$$

- For the rectangular coordinate system

$$
\operatorname{Im}\left\{\mathrm{e}^{-\mathrm{j} \delta_{\mathrm{BT}}^{\mathrm{tar}}}\left(\overline{\mathbf{V}}_{\mathrm{BT}} \overline{\mathbf{V}}_{i}^{*}\right)\right\}=0,
$$

where $\delta_{\mathrm{BT}}^{\mathrm{tar}}$ is target phase shift between $\overline{\mathbf{V}}_{\mathrm{BT}}$ and $\overline{\mathbf{V}}_{i}$, and

$$
\delta_{\mathrm{BT}}^{\mathrm{tar}}=\pi / 2 \text {, }
$$

or

$$
\delta_{\mathrm{BT}}^{\mathrm{tar}}=-\pi / 2 .
$$

\section{DESCRIPTION OF THE INVESTIGATIONS}

\section{A. Assumptions}

The investigations are so planned to ensure representativeness of taken into account cases of realisation of the state estimation in a test system.

Assumptions for the described investigations are as follows:

1. The IEEE-14-bus test system is used [23].

2. One takes into considerations the test system with the quadrature booster and this system without the quadrature booster.

3. Impedances $\overline{\mathbf{z}}_{\mathrm{ET}}$ and $\overline{\mathbf{z}}_{\mathrm{BT}}$ in the model of the quadrature booster are as follows [24]:

$$
\left\{\begin{array}{l}
\overline{\mathbf{z}}_{\mathrm{ET}}=\mathrm{j} 0.05 \mathrm{pu}, \\
\overline{\mathbf{z}}_{\mathrm{BT}}=\mathrm{j} 0.05 \mathrm{pu} .
\end{array}\right.
$$

4. If in the test system, there is a quadrature booster then it is on the line between bus 5 and bus 4 , at bus 5 .

5. 11 load variants are considered. For the given variant, each active and reactive load and also power injection is defined as

$$
W=0.5 W_{b}+l \times W_{b},
$$

where $W, W_{b}$ are the calculated and base values of the mentioned quantity; $l \in\{0,0.1,0.2, \ldots, 1\} . V^{0.5+l}$ stands for the variant associated with $l$.

6. If in the test system, there is a quadrature booster, then for each load variant, the magnitude of $V_{B T}$ is defined as: $V_{B T}=0.01,0.02,0.03, \ldots, 0.2$, and phase shift $\delta_{B T}-\delta_{i}$ is equal to $-90^{\circ}$ or $90^{\circ}$.

7. One takes into account four levels of data redundancy (defined as the ratio of a number of Measurement Data (MD) and a number of state variables), i.e.: $r_{1}, r_{2}, r_{3}$, and $r_{4}$, which correspond to the following numbers of MD: $m_{1}=34, m_{2}=53, m_{3}=68$ and $m_{4}=104$, respectively. The considered levels of the data redundancy are as follows: (i) the State Estimation for the Power System without the quadrature booster (SE_PS): $r_{1}=1.17, \quad r_{2}=1,83$, $r_{3}=2.34, r_{4}=3,59$; (ii) the State Estimation for the Power System with the Quadrature Booster (SE_PS+QB): $r_{1}=1.03, r_{2}=1,61, r_{3}=2.06, r_{4}=3,15$. 
8. For each level of the data redundancy, 100 different locations of measurement systems is randomly generated. 9. Each item of MD is burden with a small error characterized by the Gaussian distribution with a mean equal to zero and standard deviation $\sigma$, defined as [25], [26]:

- for active power

$$
\sigma=1 / 3[(0.001+0.0025) F S+0.02 M]
$$

- for reactive power

$$
\sigma=1 / 3[(0.001+0.005) F S+0.02 M]
$$

- for voltage magnitude

$$
\sigma=1 / 3[(0.0005+0.0025) F S+0.003 M]
$$

where $F S$ is a measurement scope, $M$ is a measured value. 10. The state estimation is the weighted least squares one. 11. A state vector is found solving the normal equations. 12. To show changes of properties of the state estimation when in the test system the quadrature booster is installed, compared to the state estimation for the test system without the quadrature booster, for both cases the investigations are made for the same load variants, levels of the data redundancy, locations of measurement systems and characteristics of small errors burdening MD.

13. For one location of measurement systems in the test system without the quadrature booster, 100 cases of generation of small errors burdening MD is considered.

14. To investigate impact of quadrature-booster losses on properties of the state estimation one performs estimation calculations for:

$$
\left\{\begin{array}{l}
\overline{\mathbf{z}}_{\mathrm{ET}}=(0.01+\mathrm{j} 0.05) \mathrm{pu} \\
\overline{\mathbf{z}}_{\mathrm{BT}}=(0.01+\mathrm{j} 0.05) \mathrm{pu}
\end{array}\right.
$$

i.e. when real parts of $\overline{\mathbf{z}}_{\mathrm{ET}}$ and $\overline{\mathbf{z}}_{\mathrm{BT}}$ are not equal to zero. Further, SE_PS + QB, R stands for the state estimation for the test system with the quadrature booster, when the quadrature-booster losses are taken into account.

\section{B. Results of Investigation when Quadrature-Booster Losses are Neglected}

Results of the investigations are presented in Table ITable VI and Fig. 4-Fig. 9. In Table I, Table III, Table V, indices, describing properties of the state estimation for different cases, are characterized using such parameters as the minimum, maximum, mean values and standard deviation. TABLE II contains numbers of iterations for which, there are the largest numbers of cases of the state estimation, In TABLE IV and TABLE VI, for such indices as the condition number and ratio $J_{e} / J_{m}$, there are given intervals of values that the considered indices have in the largest number of cases of the state estimation. Further, those intervals are called as the-Largest-Number-of-Cases Intervals (LNC_Is). Analyzing differences of the parameters of the considered indices for SE_PS+QB and for SE_PS, their relative values are taken into account. Those relative values are calculated using

$$
p_{r \%}=100 \times\left(p_{Q B+}-p_{Q B-}\right) / p_{Q B-},
$$

where $p_{\mathrm{r} \%}$ is a relative difference of values of considered parameter $p$ for SE_PS+QB and SE_PS; $p_{\mathrm{QB}+}, p_{\mathrm{QB}-}$ are values of parameter $p$ for SE_PS+QB and SE_PS respectively.

In each of figures Fig. 4-Fig. 9, each curve presents values of the considered index sorted in order of decreasing. For a selected point of a curve, in any figures Fig. 4-Fig. 9, an ordinate is the value of the considered index to be not larger than other values determined for cases, of which number is given by abscissa (expressed as a percentage of all cases)

Fig. 4, Fig. 6, Fig. 8 refer to load variant $V^{0.5}$, and Fig. 5, Fig. 7, Fig. 9 - to load variant $V^{1.5}$.

Analysing data from Table I, one can ascertain that apart from the case of the standard deviation for MD number $m_{4}$ and load variant $V^{1.5}$, for SE_PS + QB, parameters describing number of iterations are not less than for SE_PS. The results of investigations show, that for SE_PS + QB as well as SE_PS, the most often number of iterations $\left(L_{i t}\right)$ is 6 and 7 , when the load variant is $V^{0.5}$ and $V^{1.5}$, respectively. However, when SE_PS is considered, in larger number of cases, $L_{i t} \leq 6$ (for $V^{0.5}$ ) or $L_{i t} \leq 7$ (for $V^{1.5}$ ). For example, for load variant $V^{0.5}$, the number of cases, in which $L_{i t} \leq 6$ is at least 1.6 times higher for SE_PS than SE_PS + QB, independently of the MD number. For load variant $V^{1.5}$, the number of cases, in which $L_{i t} \leq 7$ is about 1.8 times higher for SE_PS than SE_PS + QB, when the MD number is $m_{1}$, and it is nearly the same in SE_PS and in SE_PS+QB, when the MD number is $m_{4}$. In effect, one can state that the situation is more favorable when SE_PS is performed than when we perform SE_PS + QB. This is reflected in Fig. 4 and in Fig. 5, as well.

\begin{tabular}{|c|c|c|c|c|c|c|c|c|}
\hline \multirow{2}{*}{$\begin{array}{c}\text { Number of } \\
\text { MD }\end{array}$} & \multicolumn{4}{|c|}{ PS without $Q B$} & \multicolumn{4}{|c|}{ PS with QB } \\
\hline & $\mathrm{m}_{1}$ & $\mathbf{m}_{2}$ & $\mathbf{m}_{3}$ & $\mathbf{m}_{4}$ & $\mathbf{m}_{1}$ & $\mathbf{m}_{2}$ & $\mathbf{m}_{3}$ & $\mathbf{m}_{4}$ \\
\hline \multicolumn{9}{|c|}{ load variant $V^{0.5}$} \\
\hline$L_{i t}$ & 6 & 6 & 6 & 6 & 6 & 7 & 6 & 6 \\
\hline $\begin{array}{c}\text { number of } \\
\text { cases, } \%\end{array}$ & 91.5 & 97.9 & 99.5 & 98.7 & 58.4 & 55.6 & 51.6 & 61.6 \\
\hline \multicolumn{9}{|c|}{ load variant $V^{1.5}$} \\
\hline$L_{i t}$ & 7 & 7 & 7 & 7 & 7 & 7 & 7 & 7 \\
\hline $\begin{array}{c}\text { number of } \\
\text { cases, } \%\end{array}$ & 83.04 & 94.77 & 97.08 & 94.78 & 47.5 & 66.05 & 84.9 & 98.55 \\
\hline
\end{tabular}

TABLE I. PARAMETERS OF THE NUMBERS OF ITERATIONS.

\begin{tabular}{|c|c|c|c|c|c|c|c|c|}
\hline \multirow{2}{*}{$\begin{array}{c}\text { Number of } \\
\text { MD }\end{array}$} & \multicolumn{9}{|c|}{ PS without QB } & \multicolumn{5}{c|}{ PS with QB } \\
\cline { 2 - 10 } & $\mathbf{m}_{\mathbf{1}}$ & $\mathbf{m}_{\mathbf{2}}$ & $\mathbf{m}_{\mathbf{3}}$ & $\mathbf{m}_{\mathbf{4}}$ & $\mathbf{m}_{\mathbf{1}}$ & $\mathbf{m}_{\mathbf{2}}$ & $\mathbf{m}_{\mathbf{3}}$ & $\mathbf{m}_{\mathbf{4}}$ \\
\hline \multicolumn{8}{|c|}{ load variant $V^{0.5}$} \\
\hline min & 5 & 5 & 5 & 5 & 6 & 6 & 6 & 6 \\
\hline max & 8 & 8 & 6 & 6 & 10 & 9 & 8 & 7 \\
\hline mean & 5.97 & 6.01 & 6.00 & 5.99 & 6.47 & 6.59 & 6.48 & 6.38 \\
\hline std.dev. & 0.29 & 0.15 & 0.07 & 0.11 & 0.61 & 0.53 & 0.50 & 0.49 \\
\hline \multicolumn{8}{|c|}{ load variant $V^{1.5}$} \\
\hline min & 6 & 6 & 6 & 6 & 6 & 6 & 6 & 6 \\
\hline max & 11 & 12 & 8 & 7 & 14 & 12 & 9 & 8 \\
\hline mean & 7.07 & 7.04 & 7.00 & 6.95 & 7.70 & 7.37 & 7.15 & 7.01 \\
\hline std.dev. & 0.47 & 0.32 & 0.17 & 0.22 & 0.92 & 0.57 & 0.36 & 0.12 \\
\hline
\end{tabular}

TABLE II. NUMBERS OF ITERATIONS FOR WHICH, THERE ARE THE LARGEST NUMBERS OF CASES OF THE STATE ESTIMATION. 
Analyses of data from Table III reveal that in each case, i.e. for each load variant and each MD number, parameters describing the condition number are always larger for SE_PS + QB than for SE_PS. It can therefore be concluded that conditionality of SEPS $+\mathrm{QB}$ is worse than conditionality of SE_PS. This conclusion is confirmed by the results shown in Fig. 6 and Fig. 7.

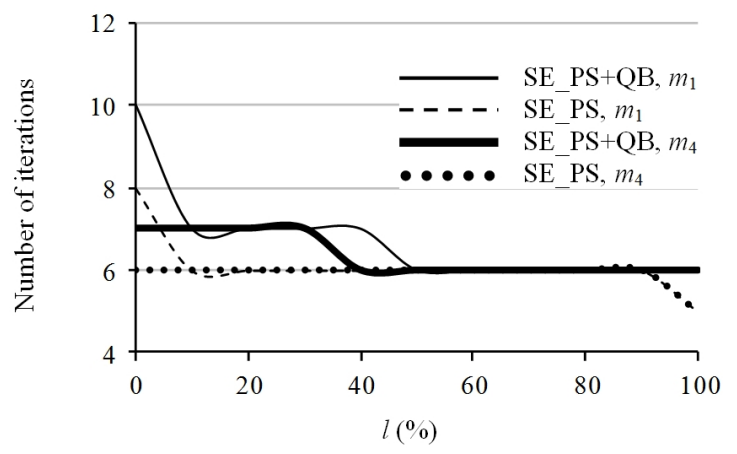

Fig. 4. The number of iterations in the state-estimation process for load variant $V^{0.5}$.

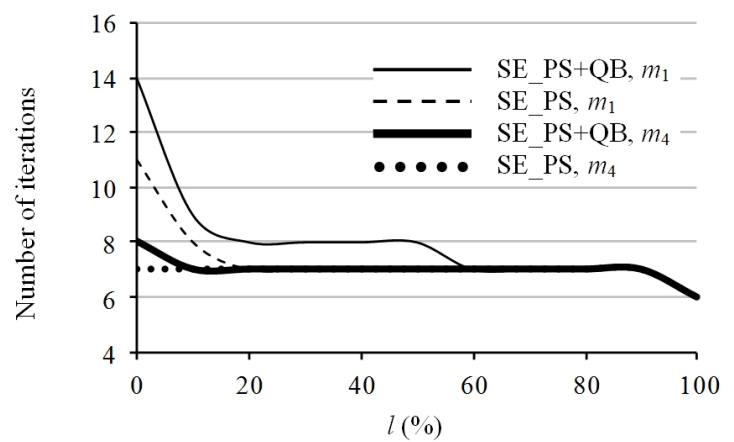

Fig. 5. The number of iterations in the state-estimation process for load variant $V^{1.5}$.

TABLE III. PARAMETERS OF THE CONDITION NUMBERS.

\begin{tabular}{|c|c|c|c|c|c|c|c|c|}
\hline \multirow{2}{*}{$\begin{array}{c}\text { Number of } \\
\text { MD }\end{array}$} & \multicolumn{4}{|c|}{ PS without QB } & \multicolumn{4}{|c|}{ PS with QB } \\
\hline & $\mathbf{m}_{1}$ & $\mathbf{m}_{2}$ & $\mathbf{m}_{3}$ & $\mathbf{m}_{4}$ & $\mathbf{m}_{1}$ & $\mathbf{m}_{2}$ & $\mathbf{m}_{3}$ & $\mathbf{m}_{4}$ \\
\hline \multicolumn{9}{|c|}{ load variant $V^{0.5}$} \\
\hline $\min$ & $2.210^{8}$ & $9.210^{7}$ & $5.710^{7}$ & $4.510^{7}$ & $8.110^{8}$ & $3.310^{8}$ & $2.110^{8}$ & $1.6510^{8}$ \\
\hline $\max$ & $5.810^{9}$ & $3.810^{8}$ & $1.310^{8}$ & $4.710^{7}$ & $2.110^{10}$ & $2.910^{9}$ & $8.710^{8}$ & $1.810^{8}$ \\
\hline mean & $3.910^{8}$ & $1.110^{8}$ & $6.110^{7}$ & $4.610^{7}$ & $2.210^{9}$ & $4.510^{8}$ & $2.310^{8}$ & $1.7110^{8}$ \\
\hline std.dev. & $5.610^{8}$ & $3.610^{7}$ & $9.010^{6}$ & $4.610^{5}$ & $2.710^{9}$ & $2.610^{8}$ & $4.910^{7}$ & $3.710^{6}$ \\
\hline \multicolumn{9}{|c|}{ load variant $V^{1.5}$} \\
\hline $\min$ & $3.210^{8}$ & $1.310^{8}$ & $8.410^{7}$ & $5.910^{7}$ & $1.310^{9}$ & $4.510^{8}$ & $2.910^{8}$ & $2.110^{8}$ \\
\hline $\max$ & $7.410^{9}$ & $1.110^{9}$ & $5.010^{8}$ & $7.610^{7}$ & $4.510^{10}$ & $7.210^{9}$ & $2.510^{9}$ & $3.210^{8}$ \\
\hline mean & $7.810^{8}$ & $1.210^{8}$ & $1.210^{8}$ & $6.610^{7}$ & $5.210^{9}$ & $9.810^{8}$ & $4.610^{8}$ & $2.510^{8}$ \\
\hline std.dev. & $8.510^{8}$ & $1.410^{8}$ & $4.810^{7}$ & $3.710^{6}$ & $6.010^{9}$ & $7.710^{8}$ & $1.910^{8}$ & $1.710^{7}$ \\
\hline
\end{tabular}

TABLE IV. THE-LARGEST-NUMBER-OF-CASES INTERVALS OF THE CONDITION NUMBERS.

\begin{tabular}{|c|c|c|c|c|c|c|c|c|}
\hline \multirow{2}{*}{$\begin{array}{c}\text { Number } \\
\text { of MD }\end{array}$} & \multicolumn{4}{|c|}{ PS without QB } & \multicolumn{4}{|c|}{ PS with QB } \\
\hline & $\mathbf{m}_{1}$ & $\mathbf{m}_{2}$ & $\mathbf{m}_{3}$ & $\mathbf{m}_{4}$ & $\mathbf{m}_{1}$ & $\mathbf{m}_{2}$ & $\mathbf{m}_{3}$ & $\mathbf{m}_{4}$ \\
\hline \multicolumn{9}{|c|}{ load variant $V^{0.5}$} \\
\hline$(\operatorname{cond}(G))_{1}$ & $210^{8}$ & $910^{7}$ & $510^{7}$ & $410^{7}$ & $110^{9}$ & $310^{8}$ & $210^{8}$ & $110^{8}$ \\
\hline$(\operatorname{cond}(G))_{\mathrm{h}}$ & $310^{8}$ & $110^{8}$ & $610^{7}$ & $510^{7}$ & $210^{9}$ & $410^{8}$ & $310^{8}$ & $210^{8}$ \\
\hline $\begin{array}{c}\text { number of } \\
\text { cases, \% }\end{array}$ & 66.64 & 64.9 & 70.28 & 100 & 49.85 & 70.48 & 94.25 & 100 \\
\hline \multicolumn{9}{|c|}{ load variant $V^{1.5}$} \\
\hline$(\operatorname{cond}(G))_{1}$ & $410^{8}$ & $110^{8}$ & $110^{8}$ & $610^{7}$ & $210^{9}$ & $610^{8}$ & $310^{8}$ & $210^{8}$ \\
\hline$(\operatorname{cond}(G))_{\mathrm{h}}$ & $510^{8}$ & $210^{8}$ & $210^{8}$ & $710^{7}$ & $310^{9}$ & $710^{8}$ & $410^{8}$ & $310^{8}$ \\
\hline $\begin{array}{c}\text { number of } \\
\text { cases, \% }\end{array}$ & 27.66 & 64.01 & 60.33 & 81.08 & 29.93 & 26.7 & 45.38 & 99.68 \\
\hline
\end{tabular}

The values of ratio $J_{e} / J_{m}$ in TABLE $\mathrm{V}$ show that for taken into account load variants and for each MD number, the mean value of ratio $J_{e} / J_{m}$ is larger for SE PS + QB than for SE_PS. The relative difference of mean values of ratio $J_{e} / J_{m}$ for SE PS + QB and SE PS is from $2.48 \%$ to $3.61 \%$ for load variant $V^{0.5}$ and from $4.93 \%$ (for $m_{1}$ ) to $12.32 \%$ (for $m_{4}$ ) for load variant $V^{1.5}$. From the point of view of the mean value of ratio $J_{e} / J_{m}$, one can state, that the power system loads are larger, the properties of the state estimation of the power system with the phase shifter are the worse.

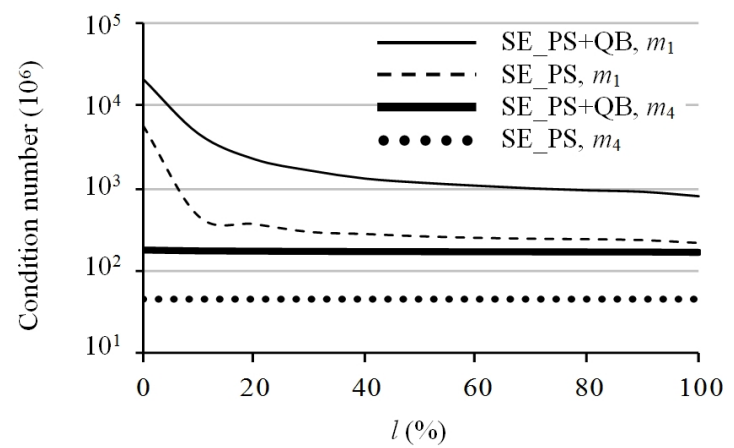

Fig. 6. The condition number in the state-estimation process for load variant $V^{0.5}$.

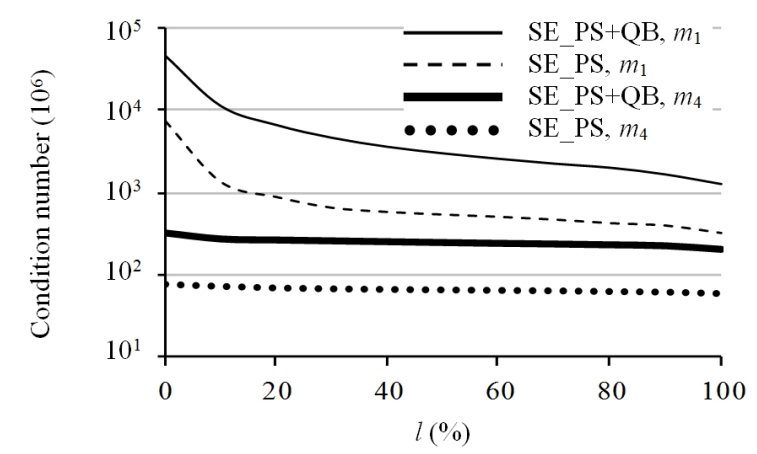

Fig. 7. The condition number in the state-estimation process for load variant $V^{1.5}$

TABLE V. PARAMETERS OF RATIOS $J_{E} / J_{M}$.

\begin{tabular}{|c|c|c|c|c|c|c|c|c|}
\hline \multirow{2}{*}{$\begin{array}{c}\text { Number of } \\
\text { MD }\end{array}$} & \multicolumn{9}{|c|}{ PS without QB } & \multicolumn{5}{c|}{ PS with QB } \\
\cline { 2 - 8 } & $\mathbf{m}_{\mathbf{1}}$ & $\mathbf{m}_{\mathbf{2}}$ & $\mathbf{m}_{\mathbf{3}}$ & $\mathbf{m}_{\mathbf{4}}$ & $\mathbf{m}_{\mathbf{1}}$ & $\mathbf{m}_{\mathbf{2}}$ & $\mathbf{m}_{\mathbf{3}}$ & $\mathbf{m}_{\mathbf{4}}$ \\
\hline \multicolumn{8}{|c|}{ load variant $V^{0.5}$} \\
\hline min & 0.41 & 0.14 & 0.11 & 0.09 & 0.45 & 0.18 & 0.16 & 0.09 \\
\hline max & 0.998 & 0.85 & 0.71 & 0.53 & 0.998 & 0.85 & 0.72 & 0.44 \\
\hline mean & 0.83 & 0.51 & 0.39 & 0.25 & 0.86 & 0.53 & 0.40 & 0.26 \\
\hline std.dev. & 0.09 & 0.10 & 0.085 & 0.06 & 0.09 & 0.10 & 0.086 & 0.06 \\
\hline \multicolumn{8}{|c|}{ load variant $V^{1.5}$} \\
\hline min & 0.37 & 0.17 & 0.11 & 0.076 & 0.49 & 0.24 & 0.14 & 0.10 \\
\hline max & 0.998 & 0.85 & 0.69 & 0.46 & 0.998 & 0.83 & 0.78 & 0.58 \\
\hline mean & 0.83 & 0.50 & 0.38 & 0.24 & 0.87 & 0.54 & 0.42 & 0.27 \\
\hline std.dev. & 0.096 & 0.10 & 0.08 & 0.057 & 0.08 & 0.098 & 0.088 & 0.07 \\
\hline
\end{tabular}

Figure 8 and Fig. 9 show, that for the majority of cases, ratio $J_{e} / J_{m}$ is larger for SE PS + QB than for SE_PS, when load variant $V^{0.5}$ is taken into account, and this ratio is always larger for SE_PS+QB than for SE_PS for load variant $V^{1.5}$.

For different MD numbers, the minimum values of ratio $J_{e} / J_{m}$ for SE_PS + QB are larger than for SE_PS. The exception is the case for MD number $m_{4}$ and load variant $V^{0.5}$. For that case, the relative difference of minimum values of ratio $J_{e} / J_{m}$ for SE_PS + QB and SE_PS is $-6.52 \%$. For 
other MD numbers, that difference is from $9.5 \%$ to $54.74 \%$ for load variant $V^{0.5}$ and from $22.92 \%$ to $43.26 \%$ for load variant $V^{1.5}$.

TABLE VI. THE-LARGEST-NUMBER-OF-CASES INTERVALS OF $\operatorname{RATIOS} J_{E} / J_{M}$.

\begin{tabular}{|c|c|c|c|c|c|c|c|c|}
\hline \multirow{2}{*}{$\begin{array}{c}\text { Number } \\
\text { of } M D\end{array}$} & \multicolumn{4}{|c|}{ PS without QB } & \multicolumn{4}{|c|}{ PS with QB } \\
\hline & $\mathrm{m}_{1}$ & $\mathbf{m}_{2}$ & $\mathbf{m}_{3}$ & $\mathbf{m}_{4}$ & $\mathbf{m}_{1}$ & $\mathbf{m}_{2}$ & $\mathbf{m}_{3}$ & $\mathbf{m}_{4}$ \\
\hline \multicolumn{9}{|c|}{ load variant $V^{0.5}$} \\
\hline$\left(\boldsymbol{J}_{e} / \boldsymbol{J}_{M}\right)_{1}$ & 0.85 & 0.48 & 0.37 & 0.24 & 0.93 & 0.56 & 0.38 & 0.24 \\
\hline$\left(J_{e} / J_{M}\right)_{\mathrm{h}}$ & 0.86 & 0.49 & 0.38 & 0.25 & 0.94 & 0.57 & 0.39 & 0.25 \\
\hline $\begin{array}{c}\text { number } \\
\text { of cases, } \\
\%\end{array}$ & 4.76 & 4.17 & 4.91 & 6.53 & 5.53 & 4.03 & 4.7 & 6.48 \\
\hline \multicolumn{9}{|c|}{ load variant $V^{1.5}$} \\
\hline$\left(J_{e} / J_{M}\right)_{1}$ & 0.88 & 0.49 & 0.36 & 0.22 & 0.9 & 0.52 & 0.4 & 0.27 \\
\hline$\left(J_{e} / J_{M}\right)_{\mathrm{h}}$ & 0.89 & 0.5 & 0.37 & 0.23 & 0.91 & 0.53 & 0.41 & 0.28 \\
\hline $\begin{array}{c}\text { number } \\
\text { of cases, } \\
\%\end{array}$ & 4.72 & 4 & 4.74 & 7.56 & 5.7 & 4.25 & 5.05 & 6.68 \\
\hline
\end{tabular}

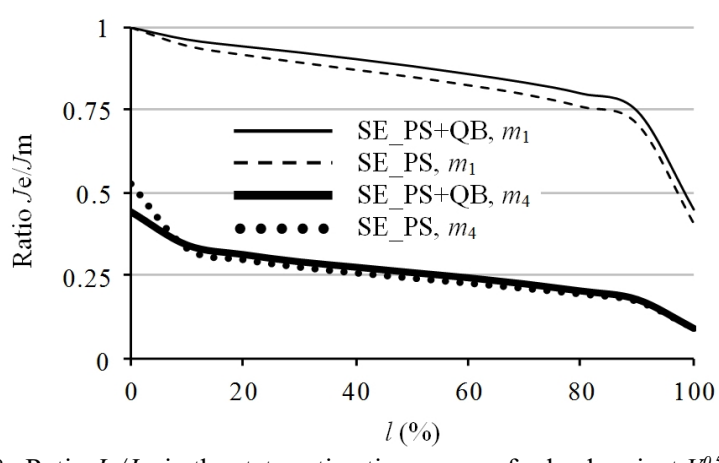

Fig. 8. Ratio $J e / J m$ in the state-estimation process for load variant $V^{0.5}$.

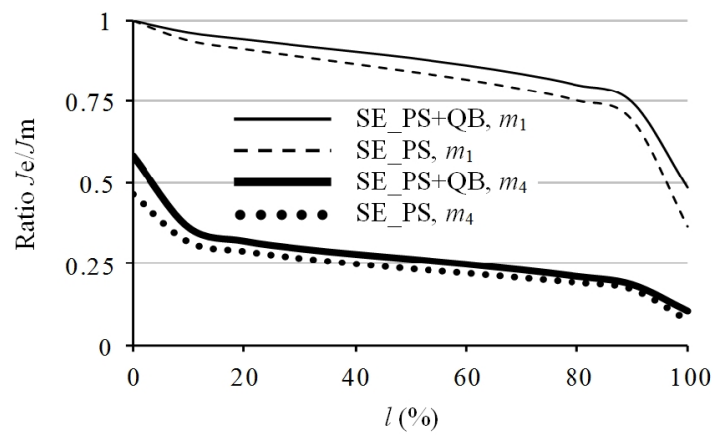

Fig. 9. Ratio $\mathrm{Je} / \mathrm{Jm}$ in the state-estimation process for load variant $V^{1.5}$.

Differences between maximum values of ratio $J_{e} / J_{m}$ for SE PS + QB and SE PS are relatively small (not more than $2 \%$ ) for MD numbers $m_{1}, m_{2}, m_{3}$ for load variant $V^{0.5}$ and for MD numbers $m_{1}, m_{2}$ for load variant $V^{1.5}$. They are noticeable for MD number $m_{4}$ for load variant $V^{0.5}$ $(-16.76 \%)$ and for MD numbers $m_{3}, m_{4}$ for load variant $V^{1.5}$ (13.08 \% and $25.86 \%$, respectively).

\section{Results of Investigation when Quadrature-Booster Losses are Considered}

Consideration of quadrature-booster losses in the estimation calculation involves changes of indices characterizing feature of the state estimation. In TABLE VII-TABLE IX, there are shown relative changes of parameters of indices: $L_{i t}, \operatorname{cond}(\mathbf{G})$ and $J_{e} / J_{m}$ calculated as follows

$$
p_{r \%}=100 \times\left(p_{\mathrm{QB}+, R}-p_{\mathrm{QB}+, X}\right) / p_{\mathrm{QB}+, X},
$$

where $p_{\mathrm{r} \%}$ is a relative difference of values of considered parameter $p$ for SE_PS $+\mathrm{QB}, \mathrm{R}$ and $\mathrm{SE} \_\mathrm{PS}+\mathrm{QB} ; p_{\mathrm{QB}+, R}$, $p_{\mathrm{QB}+, X}$ are values of parameter $p$ for $\mathrm{SE}$ - PS $+\mathrm{QB}, \mathrm{R}$ and SE_PS + QB respectively.

In Table VII-Table IX, there are results of calculation for $95 \%$ of all considered cases. A set of those cases is defined, omitting $2.5 \%$ of cases, for which the considered index has the largest values, and $2.5 \%$ of cases, for which the considered index has the smallest values.

In general, differences between mean values of indices: $L_{i t}$, cond $(\mathbf{G})$ and $J_{e} / J_{m}$ for SE_PS + QB, R and SE_PS + QB, which are given in Table VII-Table IX, are not too large. In absolute values, values of those differences are: (i) for $L_{i t}$ : not more than $0.73 \%$, (ii) for $\operatorname{cond}(\mathbf{G})$ : not more than $1.91 \%$, (iii) for $J_{e} / J_{m}$ : not more than $0.54 \%$ for load variant $V^{0.5}$ and not more than $4.87 \%$ for load variant $V^{1.5}$.

Excluding the cases for MD number $m_{4}$ and load variant $V^{1.5}$, for SE_PS + QB,R and SE_PS + QB the minimum and maximum values of $L_{i t}$, respectively, are the same. It should be also noted, that for MD number $m_{4}$ and load variant $V^{1.5}$, in the largest number of cases of SE_PS + QB as well as SE_PS + QB,R the iteration number is equal to 7. The number of such cases is about $98.5 \%$ for SE_PS + QB and about $97 \%$ for SE_PS + QB,R.

TABLE VII. PARAMETERS OF THE NUMBERS OF ITERATIONS.

\begin{tabular}{|c|c|c|c|c|c|c|c|c|}
\hline Load variant & \multicolumn{9}{|c|}{$V^{\mathbf{0 . 5}}$} & \multicolumn{5}{|c|}{$V^{\mathbf{1 . 5}}$} \\
\hline Number of MD & $\mathbf{m}_{\mathbf{1}}$ & $\mathbf{m}_{\mathbf{2}}$ & $\mathbf{m}_{\mathbf{3}}$ & $\mathbf{m}_{\mathbf{4}}$ & $\mathbf{m}_{\mathbf{1}}$ & $\mathbf{m}_{\mathbf{2}}$ & $\mathbf{m}_{\mathbf{3}}$ & $\mathbf{m}_{\mathbf{4}}$ \\
\hline min & 0.00 & 0.00 & 0.00 & 0.00 & 0.00 & 0.00 & 0.00 & 0.00 \\
\hline max & 0.00 & 0.00 & 0.00 & 0.00 & 0.00 & 0.00 & 0.00 & 14.3 \\
\hline mean & 0.25 & -0.36 & -0.73 & -0.43 & 0.16 & -0.28 & 0.18 & 0.06 \\
\hline std.dev. & 0.27 & 0.60 & -0.75 & -1.57 & -0.68 & -1.67 & 4.04 & $*$ \\
\hline *) the reference standard deviation is equal to 0.00.
\end{tabular}

TABLE VIII. PARAMETERS OF THE CONDITION NUMBERS

\begin{tabular}{|c|c|c|c|c|c|c|c|c|}
\hline \multirow{2}{*}{$\begin{array}{c}\text { Load variant } \\
\text { Number of MD }\end{array}$} & \multicolumn{5}{|c|}{$\boldsymbol{V}^{\mathbf{0 . 5}}$} & \multicolumn{4}{c|}{$\boldsymbol{V}_{\mathbf{1}}^{\mathbf{1 . 5}}$} \\
\cline { 2 - 9 } & $\mathbf{m}_{\mathbf{2}}$ & $\mathbf{m}_{\mathbf{3}}$ & $\mathbf{m}_{\mathbf{4}}$ & $\mathbf{m}_{\mathbf{1}}$ & $\mathbf{m}_{\mathbf{2}}$ & $\mathbf{m}_{\mathbf{3}}$ & $\mathbf{m}_{\mathbf{4}}$ \\
\hline $\mathbf{m i n}$ & -1.06 & -1.50 & -1.51 & -1.52 & -1.89 & -2.10 & -2.27 & -2.52 \\
\hline $\mathbf{m a x}$ & -0.42 & -2.00 & -1.41 & -0.44 & -1.68 & -1.28 & -1.37 & -1.45 \\
\hline mean & -1.21 & -1.19 & -1.16 & -1.21 & -1.71 & -1.75 & -1.84 & -1.91 \\
\hline std.dev. & -1.26 & -1.55 & 0.40 & 15.4 & -1.95 & -1.06 & -1.64 & 0.64 \\
\hline
\end{tabular}

TABLE IX. PARAMETERS OF RATIOS $J_{E} / J_{M}$.

\begin{tabular}{|c|c|c|c|c|c|c|c|c|}
\hline \multirow{2}{*}{$\begin{array}{c}\text { Load variant } \\
\text { Number of MD }\end{array}$} & \multicolumn{4}{|c|}{$V^{\mathbf{0 . 5}}$} & \multicolumn{4}{c|}{$\boldsymbol{V}_{\mathbf{1}}^{\mathbf{1 . 5}}$} \\
\cline { 2 - 10 } & $\mathbf{m}_{\mathbf{2}}$ & $\mathbf{m}_{\mathbf{3}}$ & $\mathbf{m}_{\mathbf{4}}$ & $\mathbf{m}_{\mathbf{1}}$ & $\mathbf{m}_{\mathbf{2}}$ & $\mathbf{m}_{\mathbf{3}}$ & $\mathbf{m}_{\mathbf{4}}$ \\
\hline min & -0.11 & -0.72 & 0.75 & 7.60 & -0.50 & -3.77 & -1.70 & -0.28 \\
\hline max & -0.02 & 0.20 & -1.07 & 0.64 & 0.01 & -0.57 & -5.66 & -20.7 \\
\hline mean & 0.21 & 0.25 & 0.54 & -0.30 & -0.15 & -2.39 & -3.27 & -4.87 \\
\hline std.dev. & 0.83 & 1.56 & -1.47 & -2.03 & -0.25 & -0.33 & -2.63 & -17.5 \\
\hline
\end{tabular}

\section{DISCUSSION}

Presence of a phase shifter in a power system entails grater complexity of the model of this system. A number of equations, which are taken into account in the state estimation, increases. Additional equations result from (19) and (23). Besides, equations for $P_{i}, Q_{i}, P_{k}, Q_{k}$ where $i$ and $k$ are indices of terminal buses of the branch with the phase shifter, are more complex (20), (21). The number of state variables increases. Now, in the state vector are also real and imaginary parts of voltages $\overline{\mathbf{V}}_{\text {ET }}$ and $\overline{\mathbf{V}}_{\text {BT }}$. From the stateestimation viewpoint, when configuration of measuring systems in a power system does not change, situation 
becomes worse. The ratio of the MD number and the statevariable number decreases. Consequently, there is possibility of occurrence of larger values of ratio $J_{e} / J_{m}$. Such situation is observed in the performed investigations. Only for certain number of cases, when the load variant is $V^{0.5}$ and the MD number is $m_{4}$, ratio $J_{e} / J_{m}$ is larger for SE_PS than for SE_PS + QB. However, for the mentioned load variant and the MD number, in $93 \%$ of cases, relation between values of ratio $J_{e} / J_{m}$ for SE_PS and for SE_PS + QB is other. For all considered MD numbers and load variants, mean values of ratio $J_{e} / J_{m}$ are larger for SE PS + QB than for SE_PS. In general, for different MD numbers and load variants, LNC_Is of ratio $J_{e} / J_{m}$ are in a range of larger values for SE_PS $+\bar{Q}$ B than for SE_PS. Only for load variant $V^{0.5}$ and $\mathrm{MD}$ number $m_{4}$, the considered intervals are the same for SE_PS as well as for SE_PS + QB.

In many cases, smaller value of the ratio of the MD number and the state-variable number together with greater complexity of some equations leads to larger values of condition number cond $(\mathbf{G})$ and also to worse convergence of calculation process, i.e. to the larger number of iterations. The results of investigations conform the presented ascertainments. For load variant $V^{0.5}$ as well as for load variant $V^{1.5}$ and for all considered MD numbers, the mean values of the condition numbers are larger for SE_PS + QB than for SE_PS. The same observation is for numbers of iterations in the state estimation process. LNC_Is of condition numbers are in a range of larger values for $\overline{S E}_{-} \mathrm{PS}$ + QB than for SE_PS. Iteration numbers, which are in the most number of cases of the state estimation process, are equal to 6 (with exception of MD number $m_{2}$ for load variants $V^{0.5}$ and SE_PS + QB) and 7 for load variants $V^{0.5}$ and $V^{1.5}$, respectively, for SE_PS as well as for SE_PS + QB. It should be noted, that when SE_PS $+\overline{Q B}$ is considered, for the mentioned iteration numbers, the number of cases of state estimation is lower. The iteration numbers, larger than earlier-given one, are observed in larger number of cases of SE_PS + QB than of SE_PS.

In the investigations, influence of losses of the quadrature booster on properties of the state estimation process is considered. as well. The parameters describing indices: $L_{i t}$, cond $(\mathbf{G})$ and $J_{e} / J_{m}$ change relatively little, when instead of the model of the quadrature booster without losses, the model of the quadrature booster with losses is taken into account. The consequence of the mentioned changes is not change of relations between properties of the state estimation for a power system with the quadrature booster and for a power system without this device.

\section{CONCLUSIONS}

In the paper, investigations of properties of the state estimation for a power system with the quadrature booster are presented. To ensure versatility of the research, different load conditions in the power system, different numbers of measurement data (i.e. different levels of data redundancy) and different values of small errors burdening measurement data are taken into account. The conditions of investigations are so selected to cover the space of possible power system operation cases. In the investigations, properties of the state estimation are characterized with the use of the indices: the number of iterations, the condition number of the gain matrix used in estimation calculations $(\operatorname{cond}(\mathbf{G}))$, and ratio $J_{e} / J_{m}$. The number of iterations is related with the time of realization of calculation. Condition number $\operatorname{cond}(\mathbf{G})$ is a measure of sensitivity to errors burdening data to be considered in estimation process. Ratio $J_{e} / J_{m}$ show accuracy of the state estimation. The mentioned features of the investigations, to whom the paper is devoted, are essential different from features of the investigations described in many other papers. In general, in the papers of other authors there are no results of so wide conducted research on the state estimation for a power system as it is in this paper. Usually, in those papers, there are given results of investigations for specific operation states of a power system (e.g. [12]). Also, properties of the state estimation are not investigated in so wide scope as in this paper.

The considerations in this paper reveal that conditions of performing state estimation for a power system with a quadrature booster are other than for a power system, in which there is no such the device. The paper presents results of original investigations of the properties of the state estimation for a power system with the quadrature booster. Those investigations are focused on analysis of differences between values of the considered indices characterizing properties of the state estimation for a power system with the quadrature booster and the state estimation for a power system without this shifter. With the exception of small number of cases, any of the mentioned indices takes values to be not larger than a certain value for number of cases of the state estimation which is less for a power system with the quadrature booster comparing to a power system without the quadrature booster. For each load variant and each measurement-data number, comparing the power system with and without the quadrature booster, we can state that the mean value of any of the considered indices is larger for a power system with the quadrature booster. Therefore, the conclusion from the carried out investigations is that properties of the state estimation for a power system with the quadrature booster are worse than for the power system without this phase shifter.

The investigations, to be described in the paper, are realized in simulation conditions. In these conditions, it is possible to calculate all considered indices characterizing properties of the state estimation. In real-time environment, it is possible to determine the number of iterations in a stateestimation process and condition number $\operatorname{cond}(\mathbf{G})$ but this is not possible with regard to ratio $J_{e} / J_{M}$. Ratio $J_{e} / J_{M}$ can be calculated, when real values of quantities, measured in the power system, are known. In effect, ratio $J_{e} / J_{M}$ can be only determined in the conditions of simulation. The investigations in such conditions provide valuable information for the aim of choice of the best state estimation method or for the aim of additional action to improve the properties of the investigated method.

\section{REFERENCES}

[1] M. R. Iravani, D. Maratukulam, "Review of semiconductor-controlled (static) phase shifters for power systems applications", IEEE Trans. Power Syst., vol. 9, no. 4, pp. 1833-1839, 1994. DOI http://dx.doi.org/10.1109/59.331438.

[2] T. Okon, K. Wilkosz, "Phase shifter models for steady state analysis", in Proc. 17th Int. Scientific Conf. on Electric Power Eng. (EPE), 2016, pp. 1-6. DOI: 10.1109/EPE.2016.7521831. 
[3] J. Verboomen, D. Van Hertem, P. H. Schavemaker, W. L. Kling, R. Belmans, "Phase shifting transformers: principles and applications", in Proc. 2005 Int. Conf. on Future Power Systems, 2005, pp. 1-6. DOI: 10.1109/ FPS.2005.204302.

[4] M. Eremia, Chen-Ching Liu, Abdel-Aty Edris, Advanced Solutions in Power Systems:HVDC, FACTS, and Artificial Intelligence. WileyIEEE Press, 2016, ch. 7. DOI: 10.1002/9781119175391.ch7.

[5] B. Wu, M. Narimani, High-Power Converters and $A C$ Drives. Hoboken, NJ, USA: Wiley \& Sons, Inc., 2017, ch. 5. DOI: 10.1002/9781119156079.ch5.

[6] C. Schweppe, J. Wildes, "Power system static-state estimation. Part IIII”, IEEE Trans. Power Appar. \& Syst, vol. 89, no. 1, pp. 120-135, 1970. DOI: 10.1109/TPAS. 1970.292680.

[7] J. Arrillaga, R. M. Duke, "Thyristor-controlled quadrature boosting", in Proc. Institution of Electrical Engineers, vol. 126, no. 6, pp. 493498, 1979. DOI: 10.1049/piee.1979.0117.

[8] M. Zhu, L. A. Dale, "Application and modelling of quadrature boosters for the HV transmission system", in Proc. Int. Conf. Power System Technology (POWERCON), 1998, pp. 923-927. DOI: 10.1109/ICPST.1998.729220.

[9] A. Gabrijel, B. Mihalic, "Phase-shifting transformers in a structurepreserving energy function", Electr. Power Syst. Res., vol. 74, no. 2, pp. 323-330, 2005. DOI: 10.1016/ j.epsr.2004.12.002.

[10] T. Okon, K. Wilkosz, "Comparison of weighted-least-squares power system state estimation in polar and rectangular coordinate systems", in Proc. 9th Int. Conf. Environment and Electrical Eng., 2010, pp. 140-143. DOI: 10.1109/ EEEIC.2010.5489955.

[11] T. Okon, K. Wilkosz, "WLS state estimation in polar and rectangular coordinate systems for power system with UPFC: Significance of types of measurements", in Proc. Int. Symp. Modern Electric Power Systems (MEPS), 2010, pp. 1-6.

[12] D. Qifeng, Z. Boming, T. S. Chung, "State estimation for power systems embedded with FACTS devices and MTDC systems by a sequential solution approach", Electr. Power Syst. Res., vol. 55, no. 3, pp. 147-156, 2000. DOI: 10.1016/ S0378-7796(00)00084-5.

[13] J. A. Momoh, J. Z. Zhu, G. D. Boswell, S. Hoffman, "Power system security enhancement by OPF with phase shifter", IEEE Trans. Power Sys., vol. 16, no. 2, pp. 287-293, 2001. DOI: 10.1109/59.918300.

[14] E. Acha, FACTS: Modelling and simulation in power networks. Chichester: Wiley, 2004. DOI: 10.1002/0470020164.

[15] M. Arun Bhaskar, C. Subramani, M. Jagdeesh Kumar, S. S. Dash, "Voltage profile improvement using FACTS devices a comparison between SVC, TCSC and TCPST", in Proc. Int. Conf. Advances in
Recent Technologies in Communication and Computing, 2009, pp. 890-892. DOI: 10.1109/ARTCom.2009.135.

[16] R. Navabi, H. A. Shayanfar, R. Etemad, "Adaptive fuzzy PI controller for TCPST to improve power system stability optimized by ant colony method", in Proc. Int. Conf. Electrical Eng./Electronics Comp. Telecommunications and Inf. Tech. (ECTI-CON), 2010, pp. 11961200.

[17] H. Daneshi, A. K. Srivastava, A. Daneshi, "Security constrained unit commitment with phase shifter and wind generation", in Proc. IEEE PES General Meeting, 2011, pp. 1-7. DOI: 10.1109/PES.2011.6039834.

[18] D. Menniti, A. Pinnarelli, N. Sorrentino, "Coordinated control of phase shifters in multiarea power system to improve load-frequency dynamic performance", in Proc. 16th IEEE Mediterranean Electrotechnical Conf., Yasmine Hammamet, Tunisia, 2012, pp. 241244. DOI: 10.1109/MELCON. 2012.6196423.

[19] M. T. Mekonnen, R. Belmans, "The influence of phase shifting transformers on the results of flow-based market coupling", in Proc. The 9th Int. Conf. the European Energy Market, 2012, pp. 1-7. DOI: 10.1109/EEM.2012.6254746.

[20] A. E. Hraiech, K. B.-Kilani, M. Elleuch, "Control of parallel EHV interconnection lines using Phase Shifting Transformers", in Proc. The 11th Int. Multi-Conf. Sys., Signals \& Devices (SSD), Barcelona, 2014, pp. 1-7. DOI: 10.1109/ SSD.2014.6808829.

[21] A. Abur, A. G. Exposito, Power System State Estimation. Theory and Implementation. New York-Basel: Marcel Dekker, Inc., 2004.

[22] R. Jegatheesan, K. Duraiswamy, "AC:Multi-terminal DC power system state estimation - a sequential approach", Elec. Machines and Power Systems, vol. 12, no. 1, pp. 27-42, 1987. DOI: $10.1080 / 0731356870896009$

[23] Power systems test case archive. [Online]. Available: http://www.ee.washington.edu/research/pstca/

[24] B. Xu, A. Abur, "State estimation of systems with UPFCs using the interior point method", IEEE Trans. Power Syst., vol. 19, no. 3 , pp. 1635-1641, 2004. DOI: 10.1109/ TPWRS.2004.831703.

[25] J. Dopazo, O. Klitin, G. Stagg, L. Van Slyck, "State calculation of power systems from line flow measurements", IEEE Trans. Power Appar. \& Syst., vol. 89, no. 7, pp. 1698-1708, 1970. DOI: 10.1109/TPAS.1970.292826

[26] J. Dopazo, O. Klitin, L. VanSlyck, "State calculation of power systems from line flow measurements, Part II", IEEE Trans. Power Appar. \& Syst., vol. 91, no. 1, pp. 145-151, 1972. DOI: 10.1109/TPAS.1972.293324 